\title{
Studies on Time Profiles of EAS Particles Observed with the Telescope Array Surface Detectors
}

\author{
N. INOUE ${ }^{\star, a}$, R. Abe ${ }^{a}$, R. Cady ${ }^{c}$, M. Fukushima ${ }^{b}$, J.N. Matthews ${ }^{c}$, T. Nonaka ${ }^{b}$, \\ H. Sagawa ${ }^{b}$ and T. Suzawa ${ }^{a}$ for the Telescope Array Collaboration ${ }^{\dagger}$ \\ ${ }^{a}$ Graduate School of Science and Engineering, Saitama University, Saitama, Japan \\ ${ }^{b}$ Institute for Cosmic Ray Research, University of Tokyo, Kashiwa, Japan \\ ${ }^{c}$ Department of Physics, University of Utah, Salt Lake City, Utah, USA \\ E-mail: ninoue@mail.saitama-u.ac.jp
}

\begin{abstract}
The arrival time distributions (time profile) of extensive air shower secondary particles have been studied in an energy region $>10^{18} \mathrm{eV}$ with the data collected by the Telescope Array surface detectors. We present the average shapes of time profiles in ranges of primary particle energy, zenith angle and core distance. This is a phenomenological study of air shower disc structures (typically, the thickness of air shower disc) in the Ultra-High-Energy region. In addition, "A rise time" parameter derived from the time profile has been used to evaluate the stage of shower development. The characteristics of time profiles are introduced from the results in both the experimental data and simulation. Furthermore, the relation between time profile and shower development is discussed in this paper.
\end{abstract}

The 34th International Cosmic Ray Conference

30 July- 6 August, 2015

The Hague, The Netherlands

\footnotetext{
* Speaker.

$\dagger$ full author list and acknowledgments at :

http://www.telescopearray.org/images/papers/ICRC2015- authorlist.pdf
} 


\section{Introduction}

The Ultra-High-Energy (UHE) Cosmic Ray (CR) research has been initiated about 40 years ago. Several experiments have been carried out, and at present Telescope Array (TA), Yakutsk[1] and Auger[2] experiments are in operation to observe Extensive Air Showers (EASs) initiated by UHECRs. At the early stage in the study of EASs, several attempts were made to investigate the longitudinal structure of the shower disc by measuring the arrival time distribution of shower particles (Bassi et al [3], Linsley and Scarsi[4]). Using a high-speed recording system, Blake et al [5] and Kamimoto et al [6] have studied the arrival time distribution of EAS muons to clarify the behavior of EAS development through the atmosphere. Furthermore, several measurements of the disc structure at large core distances have been made to examine the feasibility of Linsley's proposal of a method to detect giant EAS without a largescale detector arrangement (Teshima et al [7], Matsumoto et al [8]).

Recent studies have been carried out by the Auger experiment in an energy region $>10^{18}$ $\mathrm{eV}$ with its surface detectors. They defined the rise time parameter from the time profile of incident muon particles and concluded that the time profiles (namely, $\Delta$ parameter which was calculated from the deviation of the measured rise time from the bench mark value) have an energy dependence which was expected as a contribution of EAS development [9].

We present the detail features on the arrival time distributions of EAS charged particles in an energy region between $10^{18.2} \mathrm{eV}$ to $10^{19.6} \mathrm{eV}$ measuring with the surface detectors (SD) of Telescope Array experiment at Utah, USA. The overall arrival time distribution of EAS particles passing an SD was obtained by utilizing a high speed recording system (FADC with a resolution of $20 \mathrm{~ns}$ ). Studies on the average shape of time profiles and the rise time parameter have been carried out for EASs detected by SD and hybrid EAS events.

\section{Experiment}

\subsection{Telescope Array experiment and SD detectors}

The TA is located in the desert of Utah, USA, about $200 \mathrm{~km}$ southwest of Salt Lake City. The center of the SD array (just the position of the Central Laser Facility) is located at $39.3^{\circ}$ in Latitude, $-112.9^{\circ}$ in Longitude and $1382 \mathrm{~m}$ above sea level. The TA is the largest hybrid detector in the northern hemisphere, which consists of single SD array and three air fluorescence telescope (FD) stations, to investigate the origin and nature of UHECRs by measuring primary energy, arrival direction, mass composition and other characteristics of UHECRs. The SD array consists of 507 plastic scintillation counters each with an area of $3 \mathrm{~m}^{2}$ and the counters are deployed in the square grid with $1.2 \mathrm{~km}$ spacing, covering about $700 \mathrm{~km}^{2}$. Three FD stations on the corners of a $20.8 \mathrm{~km}$ equilateral triangle, are overlooking the SD array.

In the TA experiment, the SD array and the FD telescopes observe EASs independently. Measured events by both SDs and FDs provide crucial data in studying the systematic differences of the reconstructed EAS parameters. The FD telescopes have been operating on moonless clear nights, and accordingly, the duty cycle of FDs is expected to be about $10 \%$, whereas that of the SD is almost $100 \%$. The wave form signal from each SD Photo-Multiplier (PMT) is processed by two channels of 12-bit FADCs with $50 \mathrm{MHz}$ sampling and FPGA for fast signal processing. When signals from two PMTs of the SD exceed 0.3 single muon peak, the 


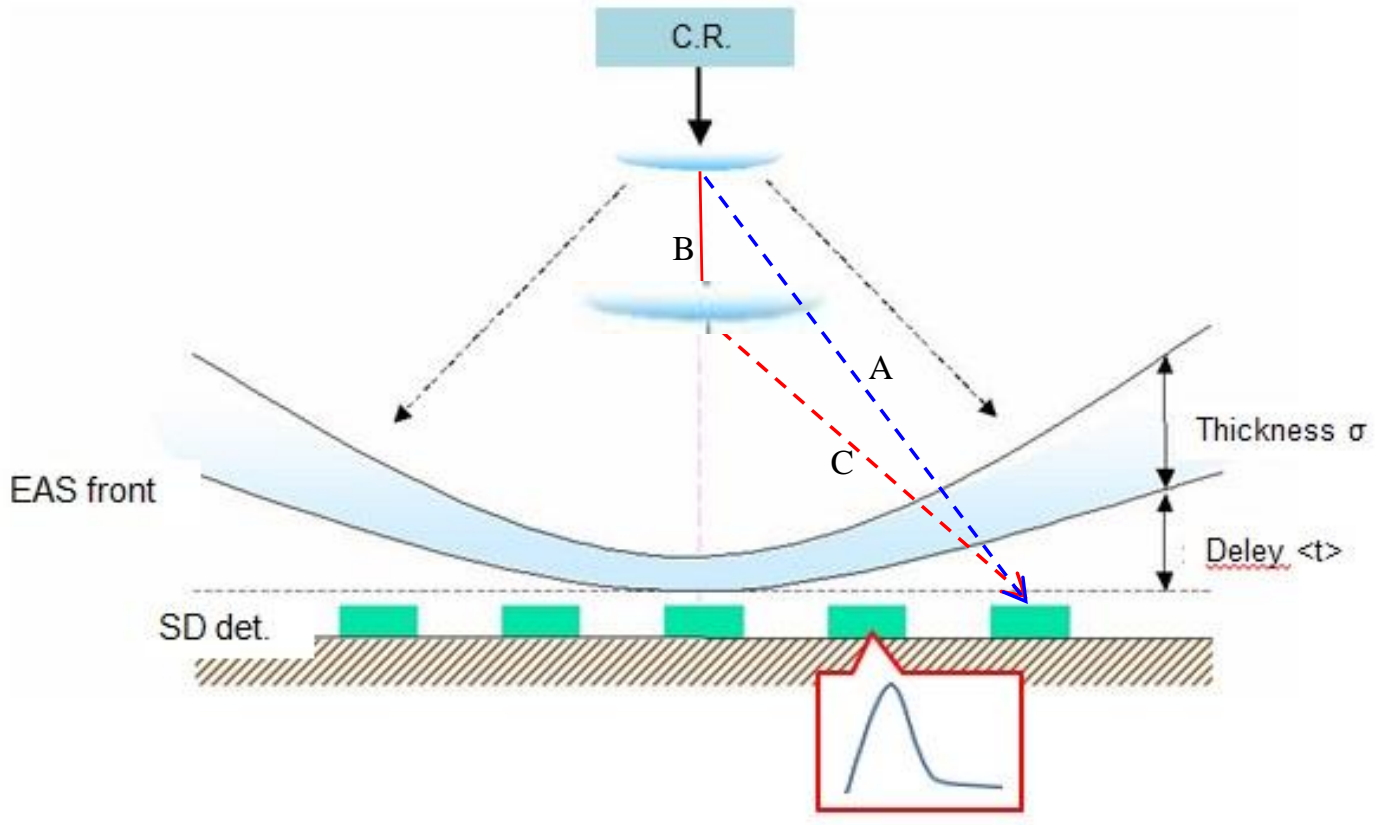

Fig. 1. An image of time profile of secondary charged particles in EAS disc.

Level-0 trigger is generated and the PMT wave form is recorded in a local memory with a time stamp by a GPS. When both PMT signals exceed a trigger threshold of three equivalent muons, the timing information is locally stored in a trigger list (Level-1 trigger). Event trigger has been implemented when a coincidence trigger among 3 neighboring SDs is produced with a threshold of three equivalent muons within 8 micro seconds.

\section{Analysis}

\subsection{Experimental data}

Experimental data of SD counters utilized in this analysis has been taken from May 2008 to May 2014. 20993 EAS events were processed in the primary energy region of $E_{0}>10^{18.0} \mathrm{eV}$ and zenith angle $(\theta)$ of $<45^{\circ}$. Each SD counter records the time profile of incident charged particles as the time profile of ADC counts with a resolution of $20 \mathrm{~ns}$ (a time unit of t.u.). Figure 2(a) shows the examples of time profiles measured by 3 SDs in different core distances ( $R$ of $850 \mathrm{~m}, 880 \mathrm{~m}$ and $1190 \mathrm{~m}$ from the top panel) for EAS with $E_{0}$ of $7 \times 10^{19} \mathrm{eV}$ and $\theta$ of $36^{\circ}$. The rise time $t_{10-50}$ is defined as the time between $10 \%$ level and $50 \%$ level in the integrated time profile. To evaluate $t_{10-50}$, FADC counts are integrated till 256 t.u. $(=5.12 \mu \mathrm{s})$. The procedure to estimate $t_{10-50}$ is shown in Figure 2(b) as an example.

Utilized SD data has been selected within the regulation of minimun $R$ and maximum $R$ to reject the SD data with the saturated output signals and to avoid a detection bias due to the threshold particles of SD at larger $R$. Available $R$ regions have been examined for each $E_{0}$ and $\cos \theta$ range. In a typical case, SD data for EAS with $E_{0}$ of $10^{18.7} \mathrm{eV}-10^{19.2} \mathrm{eV}$ and $\cos \theta>0.9$ are selected within $R$ of $450 \mathrm{~m}-1200 \mathrm{~m}$. 

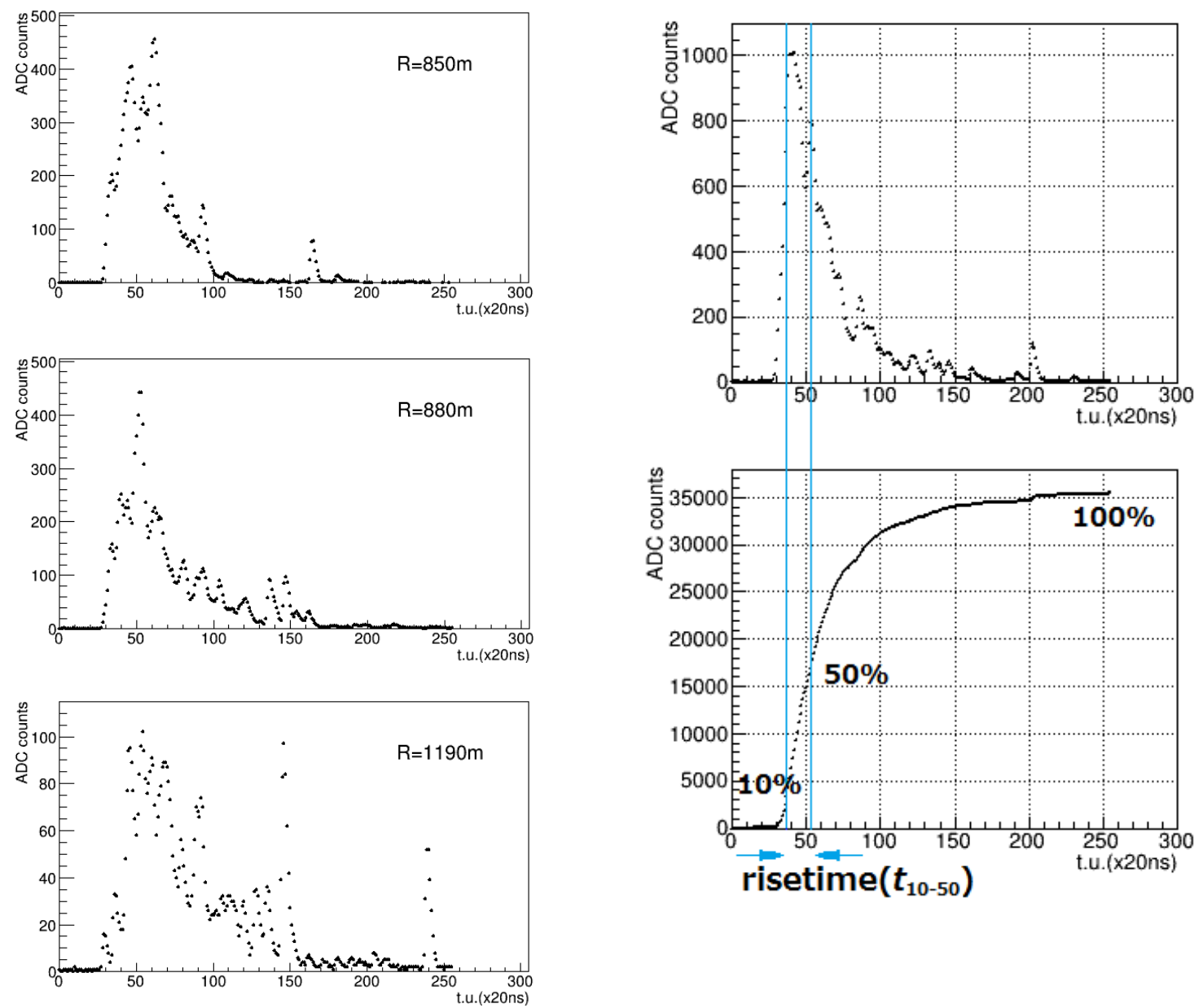

Fig. 2(a). Examples of time profiles measured by 3 SDs in different core distances ( $R$ of 850 $\mathrm{m}, 880 \mathrm{~m}$ and $1190 \mathrm{~m}$ from the top panel).

Fig. 2(b). The procedure to estimate the rise time $t_{10-50} . t_{10-50}$ is defined as the time between $10 \%$ and $50 \%$ level in the integrated time profile. An observed time profile and an integrated one are shown in an upper and a lower panel, respectively.

\subsection{Simulation data}

Simulation data has been also accumulated by the CORSIKA air-shower simulation code with QGSjet II -03 hadron interaction model and GEANT4 detector simulation code, in order to compare experimental results with ones from Monte Carlo (MC) simulation. Primary protons and irons are assumed in the EAS simulations with $E_{0}>10^{18.0} \mathrm{eV}$ and $\theta<45^{\circ}$.

\section{Results and discussion}

\subsection{Average time profile}

Average time profiles are shown in different $R$ regions of (a)400 m - $600 \mathrm{~m}$, (b)600 m $800 \mathrm{~m}$ and (c) $800 \mathrm{~m}-1000 \mathrm{~m}$ for EASs with $E_{0}$ of $10^{18.7} \mathrm{eV}-10^{19.2} \mathrm{eV}$ and $\cos \theta>0.9$ in Figure 3. In the Figures, fitted lines with a function of

$$
f(t)=a *\left(\frac{b-t}{t_{0}} \exp \left(\frac{b-t}{t_{0}}\right)\right)
$$




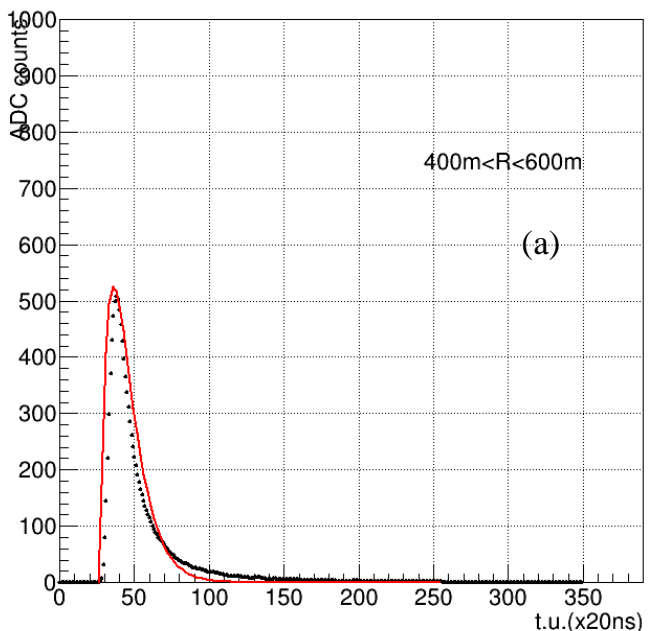

are also indicated with red lines. The width of time profiles become to be wider with $R$ because secondary particles of electromagnetic and muon components are widely distributed in time at larger $R$ due to the scattering in the atmosphere. In addition, the path differences of secondary particles due to the starting positions along the EAS axis, such as a difference between a path of $\mathrm{A}$ and a path of $\mathrm{B}+\mathrm{C}$, determines the time width at different $R$ as simply shown in Figure 1 . The width of average time profile can be estimated by a parameter of $t_{\mathrm{o}}$,

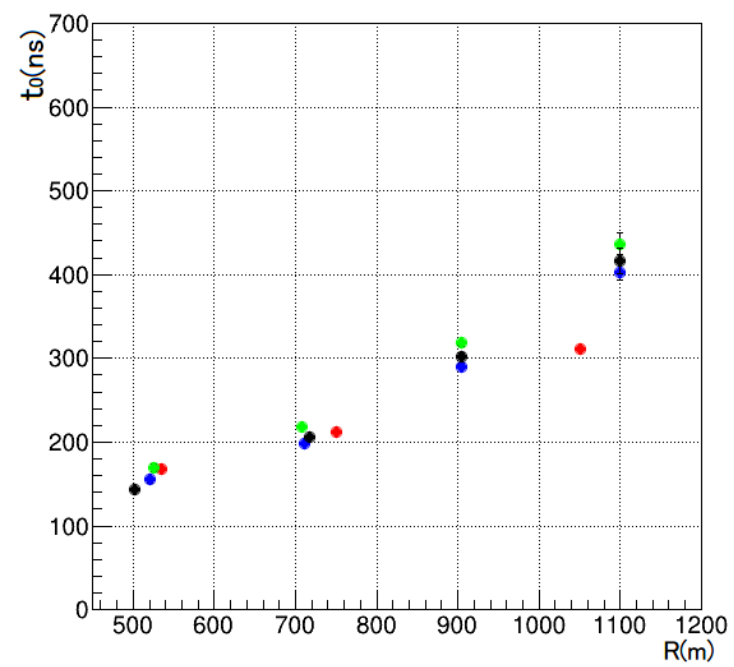

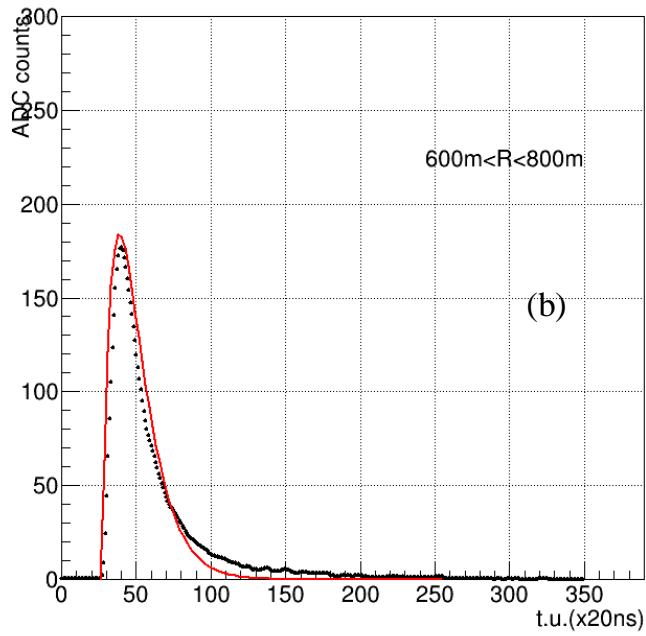

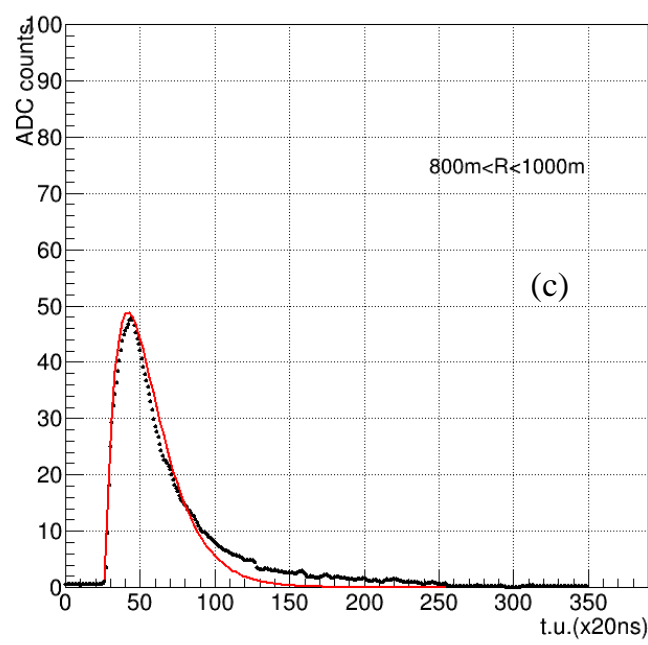

Fig. 3. Average time profiles in different $R$ regions of (a)400 m - $600 \mathrm{~m}$, (b)600 m - 800 $\mathrm{m}$ and (c) $800 \mathrm{~m}-1000 \mathrm{~m}$ for EASs with $E_{0}$ of $10^{18.7} \mathrm{eV}-10^{19.2} \mathrm{eV}$ and $\cos \theta>0.9$.

Fig. 4. $R$ dependence of $t_{\mathrm{o}}$ in a function.

Black circles: experimental data.

Green and blue circles: proton and iron MC simulation, respectively.

Red circles: previous AGASA result.

therefore $R$ dependence of $t_{\mathrm{o}}$ is shown in Figure 4 by black circles. Green and blue circles denote proton and iron MC simulation, respectively, and the previous AGASA result [10] is also 
plotted by red circles in the figure. The value of $t_{\mathrm{o}}$ clearly depend on $R$. The TA results are almost consistent with the proton and iron MC simulations, and also with the AGASA result.

\subsection{Studies on rise time}

\subsubsection{Core distance dependence}

The values of rise time $t_{10-50}$ derived from observed time profiles have been analyzed in different values of $R, E_{0}$ and zenith angle $\theta$. Average $t_{10-50}$ for EASs with $E_{0}$ of $10^{18.7} \mathrm{eV}-10^{19.2}$ $\mathrm{eV}$ and $\cos \theta>0.9$ as a function of $R$ is shown in Figure 5. We defined this relation as a standard result (a bench mark) to compare with ones from different $E_{0}$ and $\theta$ bins. The function which was fit to the data, was described by

$$
t_{10-50}=a(x+b)^{c}+d
$$

Here, $a=(3.15 \pm 0.69) \times 10^{-6}, \quad b=114 \pm 77, \quad c=2.65 \pm 0.03, d=146 \pm 17$

, and this function is also shown by a red line in Figure 5.

In general, the rise time $t_{10-50}$ becomes larger with $R$ as seen in Figure 5, and this tendency is understood because the arrival time distributions become wider by scattering and/or path differences of arrival particles when $R$ becomes larger.

Figure 6 shows zenith angle dependences of $t_{10-50}$ in the same $E_{0}$ region used in Figure 5. Zenith angle regions are classified by $\Delta \cos \theta$ of 0.1 between $\cos \theta$ of 0.7 and 1.0 . Zenith angle dependence can be found clearly in Figure 6, and it shows smaller $t_{10-50}$ in larger zenith angle region. This dependence can be explained as the EAS development gives a contribution to the rise time $t_{10-50}$ through the geometrical path differences between a path $\mathrm{A}$ and a path $\mathrm{B}+\mathrm{C}$ sketched in Figure 1.

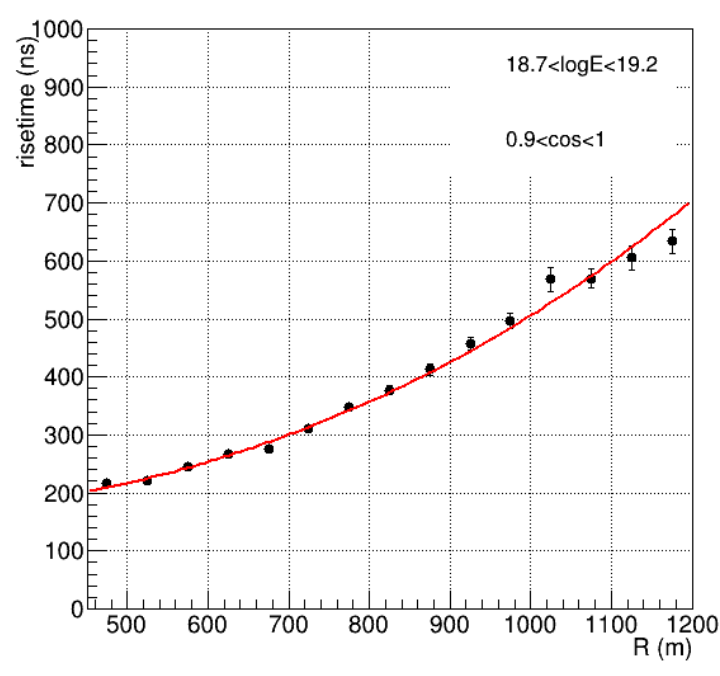

Fig. 5. Average rise time $t_{10-50}$ for EAS with $E_{0}$ of $10^{18.7} \mathrm{eV}-10^{19.2} \mathrm{eV}$ and $\cos \theta>0.9$, as a bench mark result.

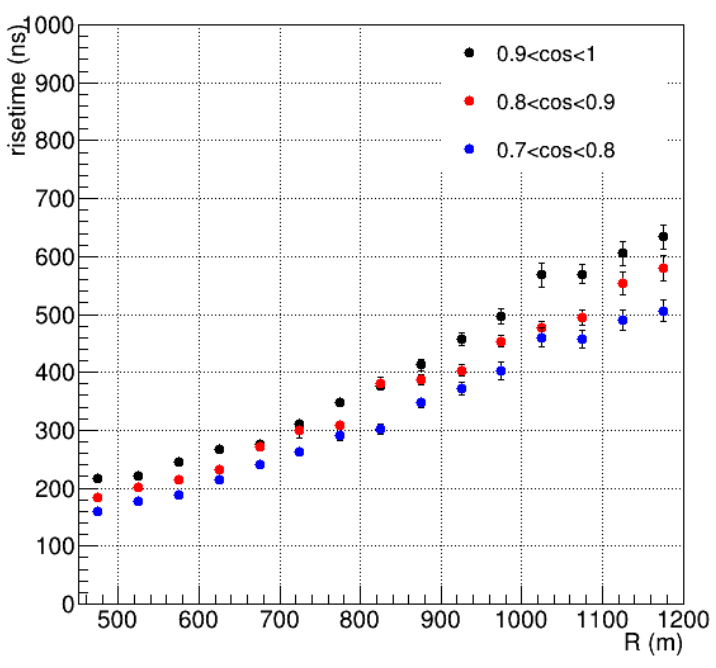

Fig. 6. Zenith angle dependences of $t_{10-50}$ in the same $E_{0}$ region used in Figure 5. Black, red and blue points show the average $t_{10-50}$ for EASs with 3 different zenith angle regions indicated in the figure. 


\subsubsection{Primary energy dependence}

Beside zenith angle dependence in $t_{10-50}$, studies on primary energy dependence are essentially important to investigate the EAS development by the use of the SDs with a duty cycle of about $100 \%$. From the result of zenith angle dependence which was caused by the contribution of different stages of EAS developments, observed $t_{10-50}$ values are also expected to be showing the energy dependence because $t_{10-50}$ becomes larger for EAS developing in deeper atmosphere. Figure 7 shows the average $t_{10-50}$ at $R$ of $800 \mathrm{~m}$ as a function of $E_{0} . t_{10-50}$ at $R$ of 800 $\mathrm{m}$ is just a value at a middle $R$ in the bench mark result as seen in Figure 5, therefore an energy dependence could be estimated in wider $E_{0}$ range. Expected results from proton and iron MC simulations are also shown in Figure 7. Experimental results are showing the weak energy dependence, and it suggests that the time profile is considered to be a useful observable by SD to study on the EAS development.

Furthermore, the energy dependence of rise time has been studied with the same method utilized by AUGER collaboration [9]. Average $t_{10-50}$ has been obtained for different $E_{0}$ regions with an interval of $\Delta \log E_{0}=0.2$ above $10^{18.2} \mathrm{eV}$. Each $t_{10-50}$ at different $R$ is compared with a bench mark function shown in 4.2.1 and the average $\langle\Delta\rangle$ is evaluated from a weighted sum of the deviations of the rise time $t^{i} 10-50$ from the bench mark value, as shown in a following formula.

$$
<\Delta\left(E_{j}<E<E_{k}\right)>=\frac{1}{N} \sum_{i=0}^{N} \frac{t_{10-50}^{i}-t_{\text {bench }}(R, \theta, E)}{\sigma_{10-50}^{i}}
$$

Here, $\sigma_{10-50}^{i}$ is the uncertainty estimated from individual $t_{10-50}$ distribution. Average $\langle\Delta\rangle$ as an $E_{0}$ dependence is shown in Figure 8 together with Auger result [9]. The correlation with $E_{0}$ could be found in Figure 8, and it shows that the time profile of EAS secondary particles is a useful parameter to estimate the feature of EAS shower development in addition to $X_{\max }$ studies by FD.

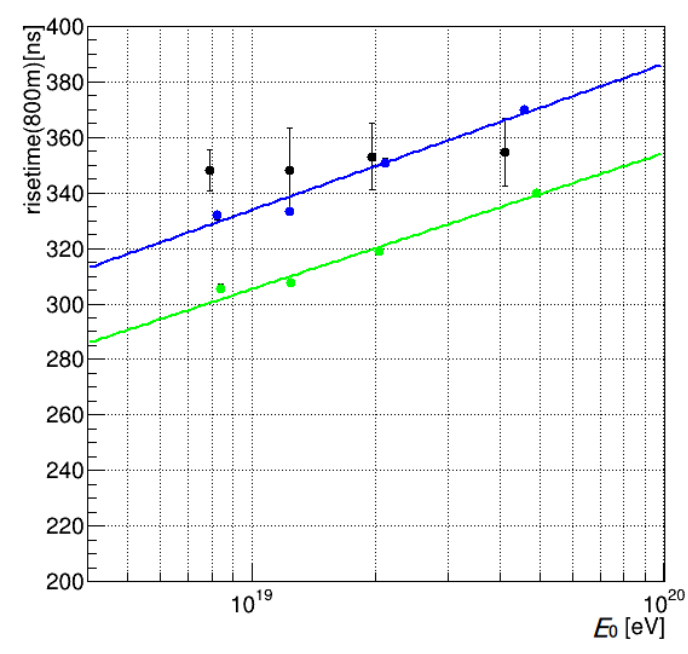

Fig.7. Average $t_{10-50}$ at $R$ of $800 \mathrm{~m}$ as a function of $E_{0}$. Proton and iron MC simulation results are also shown by blue and green points, respectively.

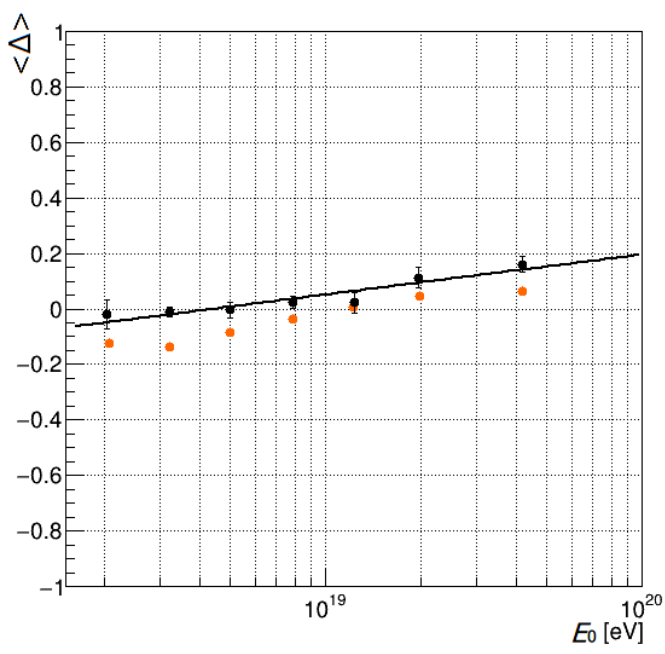

Fig.8. Black and orange points show average $\Delta(<\Delta>)$ as a function of $E_{0}$ from present analysis and previous AUGER result [9]. The line is the linear fit to the TA data. 


\subsubsection{Comparison of $\Delta$ with $X_{\max }$}

In this paper, studies on time profile have been introduced with only TA-SD data, so far. Hybrid experiment by both SD and FD detectors has been carried out in TA experiment, and we show the correlation between $X_{\max }$ and individual $\Delta$ for hybrid events with $E_{0}$ of $10^{18.2} \mathrm{eV}-10^{19.6} \mathrm{eV}$ and $\cos \theta>0.8$ in Figure 9. The data points are shown in black, and the average $X_{\max }$ for several $\Delta$ bins are shown in red circles. Positive correlation could be found in the figure, and this shows that $\Delta$ value derived from SD observation can be utilized to estimate the difference of shower development.

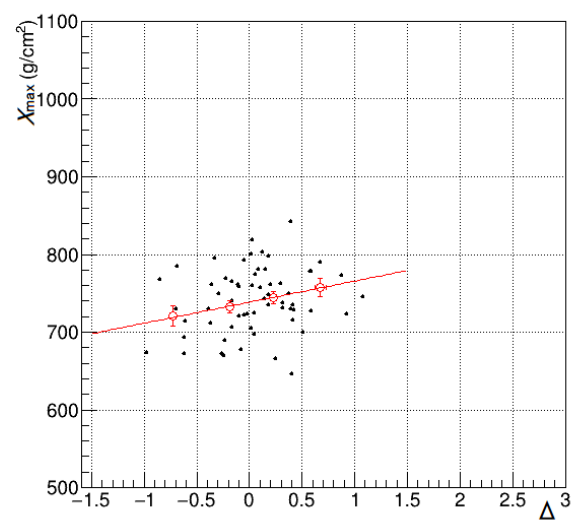

Fig. 9. The correlation between $X_{\max }$ and $\Delta$ for hybrid events with $E_{0}$ of $10^{18.2} \mathrm{eV}$ $10^{19.6} \mathrm{eV}$ and $\cos \theta>0.8$.

\section{Summary}

The time profile of EAS secondary particles is a basic observable of TA-SD with almost $100 \%$ duty cycle observation. Both electromagnetic and muon particles are mixed together in the EAS disc and their proportion changes with $R$. According to the current results, the time profile provides us a fruitful information on the EAS longitudinal development and it is consistent with the previous results by AUGER. The analysis for more hybrid events and with the rise time of $t_{5-30}$ which could be more sensitive to EAS development, will be progressed.

\section{References}

[1] V. P. Egorova, et al., Nucl. Phys. B (Proc. Suppl.) 136 (2004) p3

[2] J. Abraham, et al., Phys. Rev. Lett. 101 (2008) 061101

[3] P. Bassi, G. Clark and B. Rossi, Phys. Rev. 92 (1953)p441

[4] J. Linsley and L. Scarsi, Phys. Rev. 128 (1962)p2384

[5] P.R. Blake, D.M. Mann, W.F. Nash, B.O. O’Conell and R.B. Strutt, J. Phys. G: Nucl. Phys. 8 (1982) p1605

[6] F. Kakimoto, T. Kaneko, Y. Mizumoto, K. Suga, N. Inoue, K. Nishi, Y. Yamada, N. Tajima, E. Gotoh, H. Nakatani, H. Yoshii, R. Anda, C. Aguirre, P.K. MacKeown, K. Murakami, T. Hara, Y. Toyoda and T. Maeda, J. Phys. G:Nucl. Phys. 9 (1983) p339

[7] M. Teshima, Y. Matsubara, T. Hara, N. Hayashida, M. Honda, F. Ishikawa, K. Kamata, T. Kifune, M. Mori, M. Nagano, K. Nishijima, H. Ohoka, Y. Ohno and G. Takahashi, J. Phys. G: Nucl. Phys. 12 (1986) p1097

[8] H. Matsumoto, A. Iyono, I. Yamamoto, M. Kohata, K. Okei, S. Tsuji, T. Nakatsuka and N. Ochi, Nuclear Instrumental and Method in Physics Research, A 614 (2010) p475-482

[9] H. Wahlberg for the Pierre Auger Collaboration, Proc. of the $31^{\text {st }}$ International Cosmic Ray Conf., HE1.4 (2009)

[10] M. Takeda, N. Sakaki, K. Honda et al., Astropart. Phys. 19 (2003) p447-462 